\title{
TÉLÉDÉTECTION DES ÉLÉMENTS SEMI-NATURELS : UTILISATION DES DONNÉES PLÉIADES POUR LA DÉTECTION DES HAIES
}

\author{
Mathieu Fauvel ${ }^{1,2}$, Carole Planque ${ }^{1,2}$, David Sheeren ${ }^{1,2}$, Mauro Dalla Mura $^{3}$ \\ 1 : Université de Toulouse, INP-ENSAT, UMR 1201 DYNAFOR, France \\ 2 : INRA, UMR 1201 DYNAFOR, France \\ 3 : Grenoble INP, GIPSA-Lab, France
}

\begin{abstract}
Résumé
La détection des haies à partir d'images Pléiades est abordée dans cet article. Un système de détection en deux étapes est proposé. Il est basé sur l'utilisation conjointe de l'information spatiale et de l'information spectrale. Tout d'abord, les éléments ligneux sont détectés en utilisant l'information spectrale. Puis, l'orientation locale de chaque pixel est calculée à l'aide de filtres directionnels. Les expériences menées sur une image multispectrale Pléiades acquise en septembre 2013 montrent une bonne performance de l'approche proposée (> $90 \%$ de pixels bien classés) et permettent d'envisager une détection du réseau de haies sur de grandes étendues.
\end{abstract}

Mots clés : Pléiades, haies, détection, orientation locale.

\begin{abstract}
Detecting hedgerow is discussed in this article. A detection system in two stages is proposed. It is based on using both the spatial information and the spectral information. First, the woody elements are detected using the spectral information only. Then the local orientation of each pixel is computed by using directional filters. Experiment results on an image acquired by the Pleiades satellite show that the dection of hedgerows is possible for that kind of images over large areas.
\end{abstract}

Keywords : Pléiades, hedgerow, detection, local orientation.

\section{Introduction}

L'écologie du paysage s'intéresse à comprendre l'effet des patrons spatiaux sur la présence ou l'abondance de certaines espèces. Plus particulièrement, les chercheurs s'appliquent à modéliser la façon dont les processus écologiques sont influencés par la configuration et la composition des paysages (Gergel and Turner, 2002). Pour cela, l'identification précise et la caractérisation des types d'objets paysagers (champs agricoles, objets artificiels, eau ...) constituent un enjeu important.

Dans les paysages agricoles, il est largement reconnu que les objets tels que les " arbres hors forêt » (haies, bosquets, arbres isolés...) jouent un rôle important pour la conservation et la restauration de la biodiversité (Forman and Baudry, 1984). Ces infrastructures agro-écologiques rendent de nombreux services. Les haies sont notamment utilisées comme refuges, habitats de reproduction ou corridors pour différentes espèces animales (GrashofBokdam and van Langevelde, 2005). Elles assurent également diverses fonctions telles que le contrôle des flux physiques et chimiques (limitation des pollutions diffuses, contrôle de l'érosion) ou la protection contre le vent (effet brise-vent) (Baudry et al., 2000).

L'importance des haies est aujourd'hui reconnue à l'échelle nationale et internationale. En France, les conclusions du Grenelle de l'environnement ont souligné la nécessité de produire une carte de la Trame verte incluant les haies sur tout le territoire. Au niveau européen, certaines aides accordées dans le cadre de la Politique Agricole Commune (second pilier) imposent une surface minimale d'intérêt écologique incluant les haies (Aksoy et al., 2010).

Dans le passé, les éléments linéaires ligneux ont été cartographiés manuellement, par photo-interprétation aérienne (Morant et al., 1995). De toute évidence, cette approche est coûteuse en temps et ne convient pas pour de grandes surfaces. En outre, les petites haies ne sont pas toujours incluses dans les bases de données spatiales produites par les agences nationales de cartographie (Touzet et al., 2011). Quand c'est le cas, leurs représentations cartographiques ne correspondent pas nécessairement à la définition de l'utilisateur (Baudry et al., 2000). Par exemple, la longueur minimale pour définir un objet linéaire comme une haie peut différer selon l'espèce étudiée et sa capacité de dispersion.

Au cours des dernières années, l'extraction des haies à partir d'images satellitaires a reçu une attention grandissante. En effet, la télédétection offre une bonne solution pour l'extraction automatique sur de grandes surfaces avec une périodicité temporelle adéquate. Pour des capteurs avec une résolution spatiale fine comme Pléiades, les haies sont visuellement identifiables. En effet, en mode multispectral, chaque pixel représente un carré au sol de $2 \mathrm{~m}$ de coté après rééchantillonnage (la résolution native étant de $2.8 \mathrm{~m}$ ) et, compte tenu de l'agilité du capteur, il est possible d'obtenir au moins une date 
pour la période la plus propice à la détection des haies.

Cependant, réaliser une extraction automatique ou semi-automatique est une tâche difficile car (i) spatialement, une haie est un objet linéaire similaire à une route ou un chemin et (ii) spectralement, une haie est très semblable à la végétation arborée comme les forêts .

Les méthodes récentes abordent le problème en combinant l'information spatiale et spectrale dans le processus de détection. Traditionnellement, ces méthodes considèrent une haie comme un objet linéaire constitué d'éléments arborés. On peut distinguer 4 types d'approches. Approche pixels Les caractéristiques spatiales et spectrales sont extraites de l'image, pour chaque pixel. Le NDVI ou le résultat d'une analyse en composantes principales sur les bandes spectrales du capteur sont souvent utilisés pour extraire l'information spectrale, tandis que les informations de texture telles que l'écart-type ou l'entropie sont calculés pour caractériser le contexte spatiale (Lennon et al., 2000). Ensuite, une étape de détection est effectuée pour extraire les haies au niveau du pixel. Le principal problème de ces approches est que pour les capteurs à très haute résolution spatiale, l'information spectrale n'est pas suffisante pour détecter avec précision les haies et l'information spatiale extraite est généralement isotrope, c'est à dire qu'elle est identique pour un objet spatial orienté et pour un objet spatial non-orienté, du moins si la taille du voisinage n'est pas correctement choisie.

Approche objets L'image est d'abord segmentée pour extraire les objets spatiaux et les caractéristiques sont calculées sur les objets extraits. Ensuite, une détection est utilisée pour extraire les haies au niveau des objets (Vannier and Hubert-Moy, 2008; Sheeren et al., 2009). Cependant, l'étape de segmentation est difficile pour une image à très haute résolution spatiale en raison de la grande sensibilité de la segmentation aux paramètres d'entrée et la sous/sur-segmentation conduit à des résultats qui ne sont pas toujours concluants.

Approche multiéchelle Une analyse multiéchelle dans le domaine image est d'abord réalisée. Puis, les caractéristiques spatiales et spectrales sont extraites. Ces approches peuvent être considérées comme un compromis entre les approches pixels et objets. Dans (Stach et al., 2006) et (Tansey et al., 2009) deux méthodes de reconnaissance des haies ont été proposées à partir du logiciel eCognition. Toutefois, plusieurs paramètres de la segmentation multiéchelle sont à régler, ce qui rend ces méthodes souvent peu reproductibles ou difficiles à utiliser en pratique lorsque la zone à traiter est grande.

Approches locales Ces méthodes tentent de retrouver des objets prédéfinis dans l'image. Par exemple, les champs de Markov (Zerubia et al., 2002). Néanmoins, pour ces méthodes, un modèle (modèle statistique, fenêtre spatiale ...) doit être défini. En général, le modèle a une taille, une forme et une orientation fixée et donc un grand nombre de modèles doivent être testés pour la détection, ce qui limite par conséquent l'efficacité de telles méthodes.

Un algorithme de classification en plusieurs étapes a été proposé dans (Aksoy et al., 2010). L'algorithme de classification détecte les éléments arborés à partir de l'information spectrale (NDVI) et de caractéristiques spatiales (texture et taille) puis les haies sont extraites des forêts à l'aide d'un traitement spatial. En particulier, Aksoy et al. (2010) ont utilisé des filtres de Gabor pour extraire des informations sur la texture à différentes échelles et la taille des objets a été analysée en utilisant une granulométrie morphologique. Dans cette approche, les filtres sont isotropes (filtrage identique dans toutes les directions). Par conséquent, ces filtres n'ont pas permis de distinguer les haies des forêts, ceux-ci partageant la même information spectrale et texturale. Les auteurs ont dès lors appliqué une étape de post-traitement pour extraire les haies.

Dans cet article, nous proposons l'utilisation de filtres morphologiques directionnels pour la détection des haies. Les filtres directionnels permettent le traitement d'une image dans une direction donnée (Soille, 2003; Soille and Talbot, 2001). II est ainsi possible de conserver ou de supprimer des objets en fonction de leurs orientations et donc de discriminer des objets avec ou sans orientation principale. Cette propriété est très importante pour séparer les haies des autres structures ligneuses.

Nous avions initialement proposé d'utiliser ces filtres pour détecter les haies dans une image WorldView-2 (Fauvel et al., 2013). La méthode proposée est composée de deux étapes principales : les objets arborés sont d'abord détectés dans l'image et les haies sont ensuite séparées des forêts grâce à l'utilisation d'un indice d'orientation locale.

Dans cet article, nous montrons l'application de cette méthode sur une image Pléiades. La méthode de détection de haies est d'abord présentée en section 2 . Le calcul de l'indice d'orientation est ensuite exposé en section 3. Les résultats obtenus sur l'image Pléiades sont proposés en section 4 et comparés à ceux issus de l'image WorldView-2. Nous concluons l'article en proposant des perspectives d'enrichissement de la méthode en section 5 .

\section{Méthode de détection des haies}

Dans (Fauvel et al., 2013), nous avons proposé de séparer le problème de la détection automatique des haies en deux sous-problèmes plus simples à résoudre. La figure 1 présente la chaîne de traitement proposée. Dans une première étape, les éléments arborés sont détectés selon leur radiométrie (ici les 4 bandes spectrales l'image multispectrale). La sortie de ce détecteur est la probabilité d'appartenance à la classe Arboré. Le détecteur utilisé est un modèle de mélange Gaussien classique (GMM) (Aksoy et al., 2010). Ensuite, sur cette carte de probabilité, l'orientation locale est calculée pour chaque pixel à l'aide des filtres directionnels. L'orientation locale détermine si un pixel appartient à un objet possédant une direction principale (une haie) ou à un objet ne possédant pas de direction principale (une forêt).

L'algorithme de détection est supervisé. Des pixels de références sont nécessaires pour l'apprentissage des 


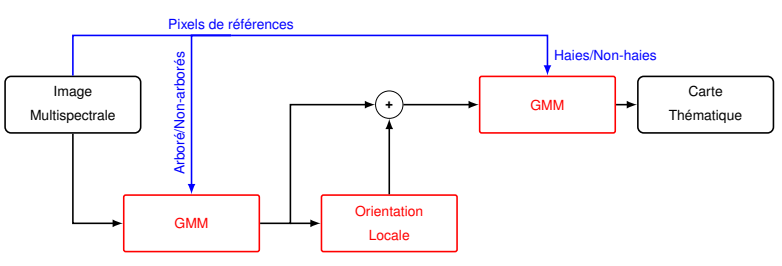

FIGURE 1 : Chaîne de traitements pour la détection des haies.

modèles statistiques. Pour cela, des pixels représentant les haies, les forêts et les non-haies/non-forêts doivent être disponibles. Dans notre cas, ces échantillons ont été sélectionnés par photo-interprétation.

\section{Orientation locale}

L'estimation de l'orientation locale fait appel à un opérateur morphologique d'ouverture et plus particulièrement, à une ouverture par chemin. Le calcul de plusieurs ouvertures par chemin permet ensuite de tracer le profil morphologique directionnel qui rend possible la séparation des haies et des forêts. Nous détaillons dans cette partie les principes de ces méthodes intégrées dans le calcul de l'orientation locale.

\subsection{Ouverture morphologique}

Une ouverture morphologique supprime complètement tous les objets de l'image qui ne contiennent l'élément structurant (un objet spatial de forme et de taille fixées). En pratique, la valeur des pixels appartenant à l'objet supprimé est modifiée par celle du premier objet le plus proche ayant une taille suffisante, c'est à dire contenant l'objet initial et l'élément structurant. La figure 2 présente un exemple sur une image synthétique.

Nous avons montré dans (Fauvel et al., 2013) que le choix de l'élément structurant était primordial. Pour la détection des haies, le choix d'un élément structurant linéaire s'impose, Figure 3. Cependant, l'utilisation d'une « ligne droite » comme élément structurant peut être trop restrictive lorsque le filtre est appliqué sur une image réelle : une haie n'est pas nécessairement rectiligne et peut présenter quelques courbures. Pour cela, nous avons proposé l'utilisation d'ouverture par chemin.

Un chemin peut être vu comme un élément structurant « flexible». Formellement, un chemin entre deux pixels est déterminé à partir d'une notion d'adjacence. L'adjacence définit l'orientation pour laquelle un pixel voisin sera inclus dans le chemin, Figure 4.

Un chemin de taille $L$ et d'orientation $\theta$ est défini comme l'ensemble des $L$ pixels adjacents. Une ouverture par chemin de taille $L$ et d'orientation $\theta$ supprime tous les objets de l'image qui ne contiennent pas le chemin. La figure 5 donne un exemple d'ouverture par chemin sur une image synthétique.

\subsection{Profil morphologique directionnel}

Le profil morphologique directionnel (PMD) est défini comme la composition de plusieurs ouvertures par

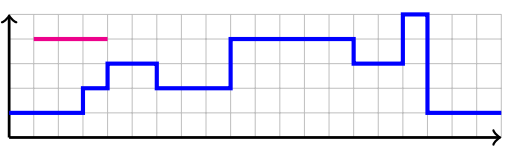

(a)

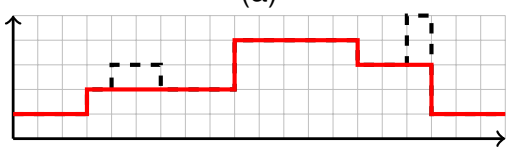

(b)

FIGURE 2 : Exemple synthétique de l'ouverture morphologique. L'axe horizontal représente les pixels et l'axe vertical représente l'intensité des pixels. (a) Image originale 1-D, où l'élément structurant est une ligne de trois pixels qui est dessiné (en magenta) dans la partie supérieure gauche de (a). (b) le résultat de l'ouverture par reconstruction est donné par la ligne rouge. La valeur des pixels des objets enlevés, en pointillés, est remplacée par celle de l'objet le plus proche avec une taille suffisante.

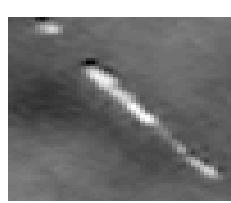

(a)

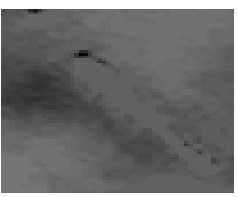

(b)

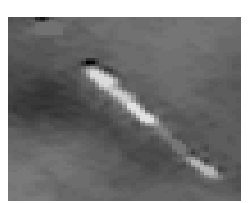

(c)
FIGURE 3 : (a) Image originale, NDVI, (b) Ouverture avec un élément structurant carré de coté 7 pixels et (c) Ouverture avec un élément structurant linéaire de taille 7 pixels et d'orientation $130^{\circ}$

chemins pour les quatre orientations possibles. II sert à distinguer les haies des forêts. La figure 6 montre deux PMD correspondant respectivement à une haie et à une forêt. Le PMD de la forêt est pratiquement plat puisque la sortie du filtre est à peu près la même quelle que soit l'orientation utilisée. Le PMD de la haie présente quant à lui une forme parabolique, indiquant que pour certaines orientations, la sortie du filtre change. En rappelant que dans le cas d'une ouverture par chemin, la valeur du pixel en sortie est soit identique ou soit diminuée, on peut voir que la structure à laquelle appartient le pixel pour la figure 6.(a) a une orientation approximativement horizontale.

A partir du PMD il est possible de calculer des indices spatiaux locaux (Soille and Pesaresi, 2002). En particulier, pour un pixel $\mathbf{x}$ donné, l'orientation locale $\mathrm{LO}(\mathbf{x})$ est définie comme la différence entre le maximum et le minimum du PMD :

$$
\mathrm{LO}(\mathbf{x})=\max _{\theta}(\operatorname{PMD}(\mathbf{x}))-\min _{\theta}(\operatorname{PMD}(\mathbf{x})) .
$$

Ainsi, un pixel appartenant à une haie aura une orien-

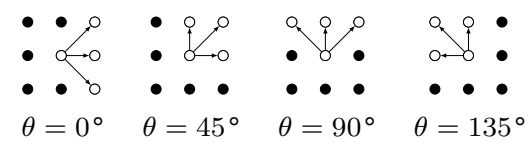

FIgURE 4 : Adjacence pour les quatre orientations possibles. Pour chaque orientation, les trois successeurs du pixel central sont compris dans un cône d'ouverture $90^{\circ}$. 


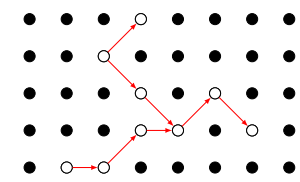

(a)

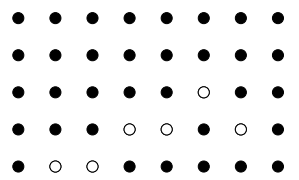

(b)
FIGURE 5 : Image binaire synthétique où les pixels blancs ont une valeur égale à 1 et les pixels noirs à 0 . (a) En rouge, tous les chemins possibles d'orientation $0^{\circ}$. (b) Résultat d'une ouverture par chemin de taille 6 pour une orientation de $0^{\circ}$.

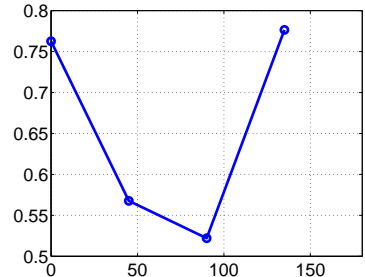

(a)

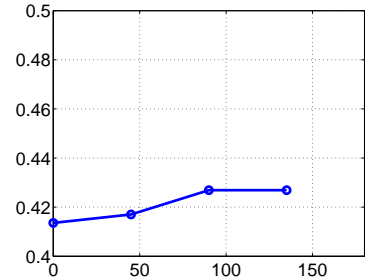

(b)
FIGURE 6 : Profil morphologique directionnel. (a) pour un pixel appartenant à une haie et (b) pour un pixel appartenant à une forêt. L'axe horizontal représente l'orientation en degré et l'axe vertical la valeur du pixel.

tation locale forte tandis qu'un pixel appartenant à une forêt aura une orientation locale proche de zéro.

La figure 7 présente l'orientation locale calculée sur la carte des probabilités d'appartenance à la classe arborée. On peut voir que tous les éléments arborés non linéaires ont une orientation locale très faible (valeurs sombres dans l'image). Ils pourront ainsi être écartés durant la dernière étape de la chaîne de traitements (étape GMM représentée sur la figure 1). Cette dernière étape consiste à détecter les haies parmi tous les objets dans l'image à l'aide du détecteur supervisé (GMM) en exploitant la la carte des probabilités d'appartenance à la classe arborée et l'orientation locale pour chaque pixel.

\section{Résultats}

\subsection{Images et données de référence}

Le site d'étude englobe plusieurs communes du canton d'Aurignac dans le Bas-Comminges, Figure 8. Se-

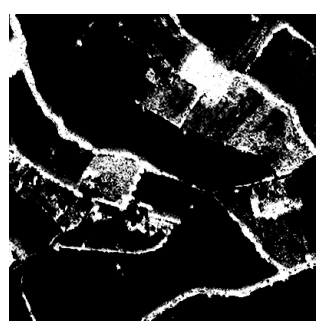

(a)

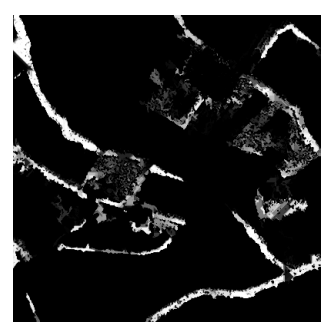

(b)
FIGURE 7 : (a) Carte des probabilités d'appartenance à la classe arborée obtenue pour l'image 1. (b) Orientation locale calculée avec les ouvertures par chemins. Pour chaque image, plus la teinte est blanche, plus la valeur associée est élevée.

\begin{tabular}{lccc}
\hline & Image 1 & Image 2 & Image 3 \\
\cline { 2 - 4 } Haie & 356 & 290 & 364 \\
Forêt & 113 & 201 & 147 \\
Non-arboré & 639 & 369 & 453 \\
\hline Total & 1108 & 860 & 964 \\
\hline
\end{tabular}

TABLE 1 : Nombre de pixels référencés pour chaque image.

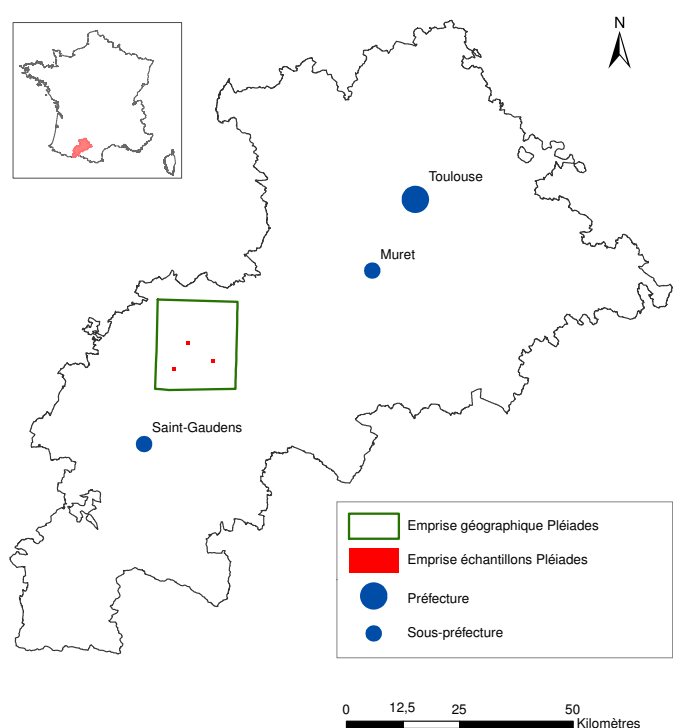

FIGURE 8 : Site d'étude et emprise des données utilisées.

Ion Sheeren et al. (2012), le paysage est vallonné, composé de cultures, de prairies, de haies et de bois et le réseau bocager est relativement bien conservé avec une densité de haies élevée, estimée à $50 \mathrm{~m} / \mathrm{ha}$ en moyenne dans le secteur.

L'étude est menée sur un couple d'images stéréoscopiques multispectrales issus du capteur Pléiades. Les données ont été acquises le 23 Septembre 2013. Trois extraits ont été utilisés pour l'analyse (figure 10).

Les pixels de référence nécessaires à l'apprentissage et à la validation ont été sélectionnés par photointerprétation. Le nombre de pixels retenu par classe est donné dans le tableau 1.

Pour comparaison, une image Worldview-2 de la même zone a été utilisée. Cette donnée a été acquise le 20 septembre 2010 , soit pratiquement à la même période que l'image Pléiades, mais avec trois ans d'ancienneté (Sheeren et al., 2012).

\subsection{Protocole expérimental}

La moitié des pixels de référence a été aléatoirement sélectionnée pour apprendre le paramètre de longueur du filtrage par chemin, le reste des pixels constituant le jeu de validation. La valeur optimale du paramètre $L$ est apprise par validation croisée sur les données d'apprentissage, pour un intervalle de valeurs testées de $[10,20, \ldots, 160]$.

Pour la détection, quatre jeux de données ont été constitués : (i) La vue 1 du couple stéréo Pléiades notée Pleiade1, (ii) La vue 2 du couple stéréo Pléiades notée Pleiade2, (iii) Les deux vues du couple stéréo Pléiades 


\begin{tabular}{lccc|c}
\hline Data & Sensibilité & Spécificité & Précision & Arboré \\
\hline Pleiade1 & 94.0 & 94.3 & 94.2 & 96.9 \\
Pleiade2 & 87.5 & 91.7 & 90.4 & 91.5 \\
PleiadeCat & 89.0 & 95.4 & 93.3 & 96.7 \\
Worldview & 83.1 & 95.3 & 91.0 & 95.1 \\
\hline
\end{tabular}

TABLE 2 : Résultats de détection pour l'image 1.

\begin{tabular}{lccc|c}
\hline Data & Sensibilité & Spécificité & Précision & Arboré \\
\hline Pleiade1 & 93.9 & 82.2 & 84.9 & 93.0 \\
Pleiade2 & 91.8 & 81.3 & 83.7 & 92.6 \\
PleiadeCat & 59.9 & 98.2 & 84.7 & 95.8 \\
Worldview & 93.5 & 92.4 & 92.8 & 98.1 \\
\hline
\end{tabular}

TABLE 3 : Résultats de détection pour l'image 2.

notée PleiadeCat, (iv) L'image Worldview-2 notée Worldview.

La précision de la détection du réseau de haies a été estimée à l'aide du jeu de validation. La sensibilité (capacité à donner un résultat positif lorsque le pixel appartient bien à une haie), la spécificité (capacité à donner un résultat négatif lorsque le pixel n'appartient pas à une haie) ainsi que la précision (capacité à donner un résultat correct) ont été évalué. La précision pour la détection des éléments arborés (qu'il s'agisse ou non d'une haie) est aussi rapportée. Les résultats sont fournis dans les tableaux 2, 3 et 4 .

\subsection{Résultats}

La figure 9 présente l'évolution de la précision estimée par validation croisée en fonction du paramètre $L$. On peut y voir que la taille moyenne de $L$ est d'environ 30 pixels ce qui correspond à environ 60 mètres si on tient compte de la résolution spatiale des données Pléiades en mode multispectral. Ainsi, pour calculer l'orientation locale, toutes les haies qui ne contiennent pas un chemin de longueur égale à 60 mètres seront filtrées et n'apparaîtront pas dans la carte de détection. Cette longueur de chemin est spécifique aux extraits sélectionnés mais est à adapter aux particularités de l'image à traiter.

Globalement, les résultats sont bons, avec des taux de précision supérieurs à 90\%, excepté pour l'image 2 avec les données Pléiades (tableaux 2, 3 et 4). Pour les images 1 et 3 , les données Pléiades donnent de meilleurs résultats, en terme de précision, que l'image Worldview2. A l'inverse c'est l'image WorldView-2 qui montre de meilleures performances pour l'image 2. L'utilisation des deux vues Pléiades (données multi-angulaires) ne permet pas d'améliorer les résultats, contrairement à (Sheeren

\begin{tabular}{lccc|c}
\hline Data & Sensibilité & Spécificité & Précision & Arboré \\
\hline Pleiade1 & 99.0 & 97.9 & 98.3 & 97.3 \\
Pleiade2 & 96.4 & 96.9 & 96.7 & 95.0 \\
PleiadeCat & 97.4 & 96.2 & 96.7 & 96.5 \\
Worldview & 96.9 & 96.2 & 96.5 & 94.0 \\
\hline
\end{tabular}

TABLE 4 : Résultats de détection pour l'image 3.

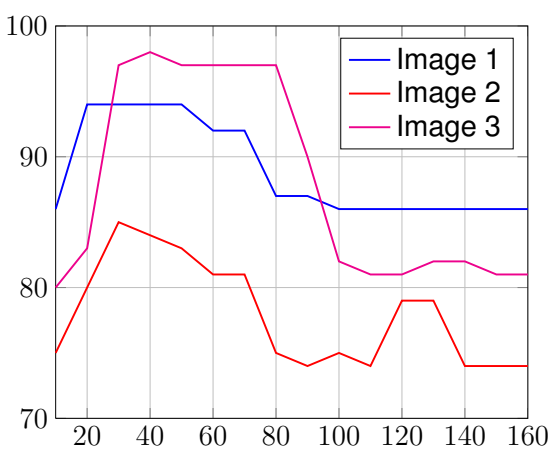

FIGURE 9 : Précisions estimées par validation croisée en fonction du paramètre $L$ des ouvertures par chemin, pour les trois images avec les données Pléiade1.

et al., 2012). Une première explication possible est le faible rapport b/h des données Pléiades en comparaison avec celui des données Worldview-2. Une autre explication possible est que le couple stéréo Pléiades présente une composante de visée latérale beaucoup plus faible que les données WorldView-2.

Les classifications sont données figure 10. Notons qu'aucun post-traitement n'a été réalisé après la détection. Visuellement, le résultat est en accord avec la précision calculée sur le jeu de validation. On retrouve beaucoup d'erreurs sur la carte de détection de l'image 2 obtenue avec Pleiade1, avec notamment une partie de la prairie détectée comme de la haie (en haut à gauche de l'image). Pour l'image 1, le résultat de la détection semble cohérent avec la majorité des haies détectées, tandis que le massif arboré n'est pas détecté. Globalement, on peut voir que les haies très fines, qu'on retrouve en haut à droite de l'image 3 ou au milieu des images 1 et 2, ne sont pas complètement détectées. Pour ces haies fines, l'ouverture par chemin est trop restrictive et ne permet pas de calculer correctement l'orientation locale. Une solution serait d'utiliser des filtres plus souples (Fauvel et al., 2014).

Quelques remarques sur la précision de la détection arboré/non-arboré. La détection est obtenue en seuillant la carte de probabilités à 0.5 : au dessus pour l'arboré, en dessous pour le non-arboré. On peut remarquer que la meilleure détection des haies est associée à la meilleure détection arboré/non-arboré. Effectivement, l'obtention d'une carte de probabilité d'appartenance fiable est primordiale pour une caractérisation précise de l'orientation locale.

\section{Conclusions et perspectives}

Une nouvelle méthode de détection des haies a été proposée dans cet article. Elle repose sur la caractérisation de l'orientation locale de chaque pixel dans l'image. Les ouvertures par chemins ont été utilisées pour calculer cette orientation locale.

Appliquée sur des données Pléiades, cette méthode fournit des résultats de détection très satisfaisants, équivalents à ceux précédemment obtenus sur une image 


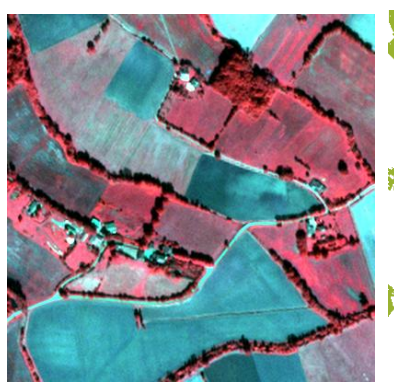

(a)

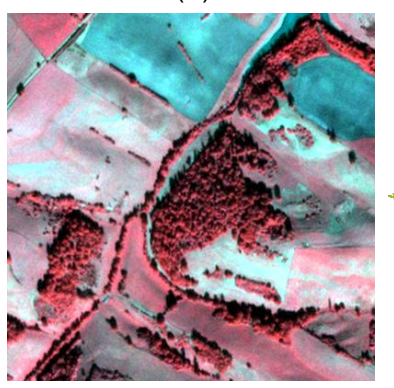

(c)

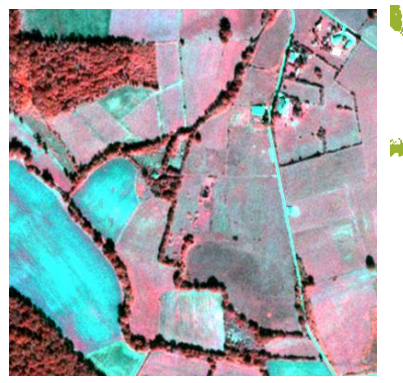

(e)

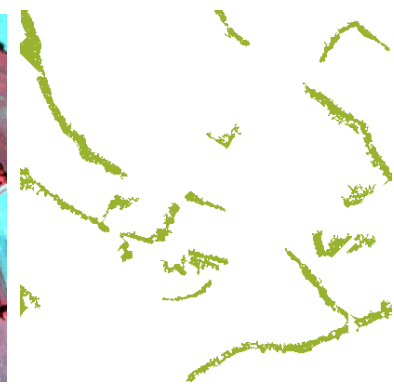

(b)

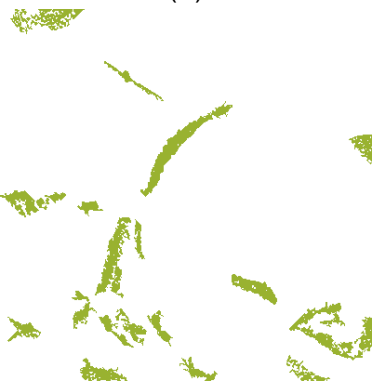

(d)

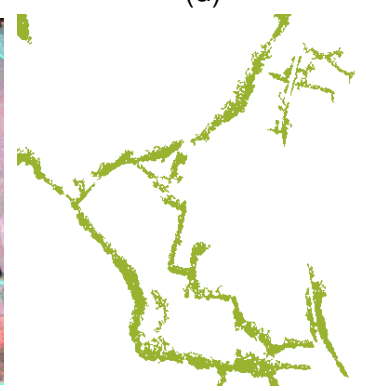

(f)
FIGURE 10 : Composition colorée des trois scènes extraites : (a) Image 1, (c) Image 2 et (e) Image 3. Carte de détection pour les images (b) 1, (d) 2 et (f) 3. obtenue avec les données Pleiade1. @ CNES 2013, distribution Airbus DS / Spot Image.

Worldview-2 de la même zone. Néanmoins, les potentialités des données Pléiades n'ont pas été exploitées dans leur entièreté. Le couple stéréoscopique acquis offre la possibilité de produire un modèle numérique de surface (MNS) qui pourrait être intégré dans la procédure d'extraction des objets en exploitant l'information de hauteur. Cette information est très discriminante pour des objets tels que les haies. Malheureusement, nous n'avons pas encore été en mesure de produire ce MNS avec un niveau de précision suffisant. II s'agit de la principale perspective d'enrichissement de ce travail.

\section{Références}

Aksoy, S., Akcay, H., Wassenaar, T., Jan. 2010. Automatic mapping of linear woody vegetation features in agricultural landscapes using very high resolution imagery. IEEE Trans. Geosci. Remote Sens. 48 (1), 511-522.

Baudry, J., Bunce, R., Burel, F., 2000. Hedgerows : An international perspective on their origin, function and management. Journal of Environmental Management 60 (1), 7 - 22.

Fauvel, M., Arbelot, B., Benediktsson, J. A., Sheeren, D., Chanussot, J., 2013. Detection of hedges in a rural landscape using a local orientation feature : from linear opening to path opening. IEEE Journal of Selected Topics in Applied Earth Observations and Remote Sensing 6 (1), 15-26.

Fauvel, M., Planque, C., Sheeren, D., Mura, M. D., Cokelaer, F., Chanussot, J., Talbot, H., July 2014. Robust path opening versus path opening for the detection of hedgerows in rural landscapes. In : Geoscience and Remote Sensing Symposium (IGARSS), 2014 IEEE International.

Forman, R. T. T., Baudry, J., 1984. Hedgerows and hedgerow networks in landscape ecology. Environmental Management 8, 495-510.

Gergel, S. E., Turner, M. G. (Eds.), 2002. Learning landscape ecology : a practical guide to concepts and techniques. SpringerVerlag, New-York.

Grashof-Bokdam, C., van Langevelde, F., 2005. Green veining : landscape determinants of biodiversity in european agricultural landscapes. Landscape Ecology (20), 7-22.

Lennon, M., Mouchot, M., Mercier, G., Hubert-Moy, L., 2000. Segmentation of hedges on casi hyperspectral images by data fusion from texture, spectral and shape analysis. In : Geoscience and Remote Sensing Symposium, 2000. Proceedings. IGARSS 2000. IEEE 2000 International. pp. 825 -827.

Morant, P., Hénaff, F. L., Marchand, J.-P., 1995. Les mutations d'un paysage bocager : essai de cartographie dynamique. Mappemonde, 5 - 8 .

Sheeren, D., Bastin, N., Ouin, A., Ladet, S., Balent, G., Lacombe, J.-P., 2009. Discriminating small wooded elements in rural landscape from aerial photography : a hybrid pixel/objectbased analysis approach. International Journal of Remote Sensing 30 (19), 4979-4990.

Sheeren, D., Masse, A., Ducrot, D., Fauvel, M., Collard, F., May, S., 2012. La télédétection pour la cartographie de la trame verte en milieu agricole. Évaluation des potentialités d'images multi-angulaires à très haute résolution spatiale. Revue Internationale de Géomatique 22 (4), 539-563.

Soille, P., 2003. Morphological Image Analysis : Principles and Applications, Second Edition. Springer, Berlin.

Soille, P., Pesaresi, M., Sep. 2002. Advances in mathematical morphology applied to geoscience and remote sensing. IEEE Trans. Geosci. Remote Sens. 40 (9), 2042-2055.

Soille, P., Talbot, H., nov 2001. Directional morphological filtering. Pattern Analysis and Machine Intelligence, IEEE Transactions on 23 (11), $1313-1329$.

Stach, N., Barnerias, C., Dommanget, A., 2006. Hedges and trees rows detection with e-cognition for the use of the french national forest inventory. In : of Salzburg, U. (Ed.), First International Conference on Object-Based Image Analysis.

Tansey, K., Chambers, I., Anstee, A., Denniss, A., Lamb, A., 2009. Object-oriented classification of very high resolution airborne imagery for the extraction of hedgerows and field margin cover in agricultural areas. Applied Geography 29 (2), 145- 157.

Touzet, T., Halbecq, X., Lecordix, F., 2011. Chaîne de production de la carte de vegetation arborée française. In : Proceedings of the 25th International Cartographic Conference.

Vannier, C., Hubert-Moy, L., july 2008. Detection of wooded hedgerows in high resolution satellite images using an objectoriented method. In : Geoscience and Remote Sensing Symposium, 2008. IGARSS 2008. IEEE International. Vol. 4. pp. IV $-731-$ IV -734 .

Zerubia, J., Jalobeanu, A., Kato, Z., 2002. Markov random fields in image processing application to remote sensing and astrophysics. J. Phys. IV France 12 (1), 117-136. 